\title{
NOUVELLE
}

\section{L'hormone anti-müllérienne : un nouveau régulateur des cellules gonadotropes hypophysaires}

\section{Implication dans le dimorphisme sexuel de l'activité gonadotrope avant la puberté} Ghislaine Garrel ${ }^{1-3}$, Chrystèle Racine ${ }^{1-3}$, David L'Hôte ${ }^{1-3}$, Chantal Denoyelle ${ }^{1-3}$, Céline J. Guigon ${ }^{1-3}$, Nathalie di Clemente ${ }^{1-3}$, Joëlle Cohen-Tannoudji ${ }^{1-3}$

\author{
${ }^{1}$ Université Paris-Diderot, Sorbonne Paris Cité, \\ Biologie fonctionnelle et adaptative (BFA), Bâtiment A Buffon, \\ 4, rue MA Lagroua Weill-Hallé, F-75013 Paris, France ; \\ ${ }^{2}$ CNRS UMR 8251, Paris, France ; \\ ${ }^{3}$ Inserm U1133, Physiologie de l'axe gonadotrope, Paris, France. \\ ghislaine.garrel-lazayres@univ-paris-diderot.fr
}

> L'hormone anti-müllérienne (AMH) ou mullerian-inhibiting substance (MIS) a été identifiée par Alfred Jost dans les années 1950 comme un facteur testiculaire clé de la différenciation sexuelle mâle, responsable de la régression des canaux de Müller chez le fœtus [1]. De nombreux travaux ont depuis démontré que l'AMH est synthétisée par les gonades mâle et femelle chez de nombreuses espèces de vertébré. Elle y contrôle de manière paracrine et autocrine la stéroïdogenèse ${ }^{l}$. L'AMH inhibe également le recrutement des follicules primordiaux dans l'ovaire de mammifères et elle est utilisée, aujourd'hui, comme un marqueur de la réserve folliculaire et de la fertilité chez la femme [1].

L'AMH appartient à la superfamille du TGF $\beta$ (transforming growth factor beta). C'est une glycoprotéine synthétisée sous la forme d'un précurseur qui se dimérise $(140$ kDa) et devient actif après avoir subi un clivage protéolytique. L'AMH se lie à un récepteur spécifique de type II (I'AMHR2) qui active, par l'intermédiaire d'un récepteur de type I (I'ALK[activin receptor-like kinase]2, 3 ou 6 ), les protéines Smad 1/5/8 qui sont impliquées dans la régulation de l'expression de gènes cibles [1].

À partir des années 2000, différents travaux ont relaté la présence du récepteur

${ }^{1}$ Processus de synthèse des hormones stéroïdes.
AMHR2 hors des gonades et notamment dans d'autres organes de la reproduction (glande mammaire, utérus et prostate) mais aussi dans le poumon fœtal et le cerveau. L'AMHR2 est en particulier exprimé dans les motoneurones de la moelle épinière, le cortex cérébral et I'hypothalamus, chez la souris [2-4]. Une seule étude témoigne de la présence d'AMHR2 dans l'hypophyse chez le rat mâle et femelle [5]. Le rôle physiologique de I'AMH dans ces différents organes demeure mal connu. Elle pourrait contribuer au dimorphisme sexuel du développement cérébral. Ainsi, il a été rapporté que l'invalidation du gène codant l'AMH chez la souris mâle est associée à une féminisation partielle du comportement d'exploration [6]. Très récemment, une étude effectuée chez la souris témoigne de l'expression de l'AMHR2 dans I'hippocampe ainsi que dans les neurones hypothalamiques, libérant la gonadotropin releasing hormone $(\mathrm{GnRH})$ qui contrôle la fonction de reproduction [7, 13] $(\rightarrow)$.

$(\rightarrow)$ Voir la Nouvelle de S. Catteau-Jonard et al., $m / s n^{\circ} 5$, mai 2016, page 441

Quel rôle pour l'AMH

dans l'hypophyse?

Ces données soulèvent donc l'idée que I'AMH pourrait participer au contrôle endocrine et neuroendocrine de la reproduction en agissant sur le complexe hypothalamo-hypophysaire. Dans
I'hypophyse, les cellules gonadotropes sécrètent, sous l'influence de la $\mathrm{GnRH}$, deux gonadotropines : la luteinizing hormone ( $\mathrm{LH})$ et la follicle-stimulating hormone (FSH). Ces deux hormones sont essentielles à la fonction de reproduction. Elles contrôlent en effet l'activité des gonades. Elles sont constituées de deux sous-unités, une sous-unité $\alpha$ qui leur est commune et une sous-unité $\beta$ qui est spécifique de chaque hormone et qui sont codées par des gènes distincts $[8,9]$.

Par des approches in vitro et aussi in vivo chez le rat, nous avons ainsi recherché si I'AMH pouvait réguler l'activité des cellules gonadotropes de I'hypophyse.

\section{Effet de l'AMH in vitro}

Nous avons dans un premier temps analysé l'action de l'AMH sur la lignée gonadotrope murine L $\beta T 2$, en testant à la fois la forme précurseur de I'AMH et la forme clivée. Nos résultats ont montré que les deux formes de I'AMH étaient capables de stimuler de manière dose-dépendante l'expression du gène Fshb (follicle stimulating hormone beta subunit). Elles agissent cependant avec des cinétiques différentes. La forme clivée agit rapidement, dès $4 \mathrm{~h}$, tandis que le précurseur atteint son effet maximal après $24 \mathrm{~h}$. Les cellules gonadotropes sont donc 

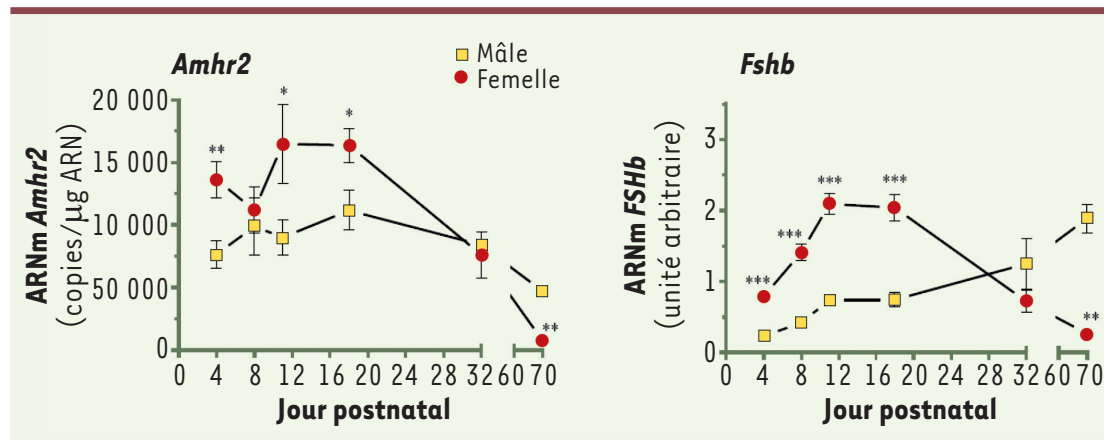

Figure 1. Ontogenèse postnatale de l'expression des gènes Amhr2 et Fshb dans l'antéhypophyse de rats mâles et femelles. Les antéhypophyses de rats mâles et femelles Wistar ont été collectées au $4^{\mathrm{e}}, 8^{\mathrm{e}}, 11^{\mathrm{e}}, 18^{\mathrm{e}}, 32^{\mathrm{e}}$ ou $72^{\mathrm{e}}$ jour postnatal. Les niveaux des transcrits ont été mesurés par PCR quantitative en temps réel et exprimés en nombre de copie/ $\mu \mathrm{g}$ d'ARN totaux pour l'Amhr2 (méthode Taqman) et en unité arbitraire pour le gène Fshb. Chaque valeur représente la moyenne \pm SEM de 6 à 13 rats. $* P \leq 0,05 ; * \star P \leq 0,01 ; * \star \star P \leq 0,001$ par rapport aux mâles du même âge. Amhr2: anti-Müllerian hormone receptor type 2 ; Fshb : follicle stimulating hormone beta subunit. SEM : erreur standard à la moyenne.

capables d'activer, par son clivage, le précurseur de l'AMH. Dans toutes les conditions testées, l'expression du gène Lhb (luteinizing hormone beta subunit) n'est pas modifiée. Ce résultat révèle que I'AMH est un des rares facteurs qui ne cible que la FSH. Elle contribuerait ainsi à la régulation différentielle des deux gonadotropines.

Son action sur l'expression de Fshb est couplée à l'activation de la voie Smad. $\varepsilon$ n effet, dans les cellules L $\beta$ T2, I'AMH induit la phosphorylation des protéines Smadl/5/8 qui sont des activateurs puissants de l'expression de Fshb [10]. Dans les lignées GH3, AtT20 et T $\alpha$ T, des cellules qui sont représentatives des autres lignages hypophysaires endocrines, le niveau d'expression de l'Amhr2 est bien plus faible (plus de vingt fois moins) et aucune activation de la voie Smad n'a été observée sous l'effet de I'AMH. L'AMH agirait donc spécifiquement sur les cellules gonadotropes de l'hypophyse.

L'AMH pourrait agir de concert avec d'autres facteurs régulateurs de la synthèse de FSH comme l'activine ou le BMP2 (bone morphogenetic protein-2) qui sont de puissants inducteurs de Fshb. Nous avons observé qu'elle potentialisait les effets de l'activine alors qu'elle inhibait celui du BMP2 sur l'expression de Fshb. Ceci démontre donc que l'AMH peut réguler la FSH hypophysaire via une interaction avec d'autres facteurs [10].

\section{Dimorphisme sexuel}

\section{de l'expression de l'Amhr2}

\section{hypophysaire chez le rat}

Afin d'évaluer s'il existe une fenêtre privilégiée d'action de I'AMH dans I'hypophyse, nous avons quantifié les ARN messagers de l'Amhr2 dans I'hypophyse chez l'adulte et au cours de la période immature de rats mâle et femelle. Nous avons observé que le nombre de copies d'ARNm Amhr2 est environ deux fois plus élevé chez la femelle que chez le mâle entre le $4^{\mathrm{e}}$ et le $18^{\mathrm{e}}$ jour post-natal (jpn) excepté à 8 jpn (Figure 1). Aucune différence n'est détectable à 32 jpn. À l'âge adulte, la situation s'inverse, la quantité d'ARNm Amhr2 devient très faible chez la femelle. De façon intéressante, ce profil est en fait très proche de celui de Fshb (Figure 1). Ces résultats témoignent donc d'un dimorphisme sexuel de l'expression de l'Amhr2 au niveau de l'hypophyse et suggèrent que I'AMH pourrait jouer un rôle important, chez la femelle, dans la période prépubertaire.

Nous avons également détecté les messagers codant I'AMH dans l'hypophyse de rats et aucune différence entre le mâle et la femelle n'a été observée au cours de la période immature. Leur présence suggère que I'AMH pourrait agir de manière paracrine/autocrine dans ce tissu. À l'âge adulte, les transcrits de I'AMH deviennent indétectables dans les deux sexes.

\section{Effet in vivo de l'AMH}

chez le rat prépubère

Pour évaluer son effet in vivo, nous avons choisi d'injecter l'AMH lors de la période immature, à un stade de fort dimorphisme sexuel de l'expression de l'Amhr2 (18 jpn). L'administration d'AMH (10 $\mu \mathrm{g}$ en injection intrapéritonéale) provoque une augmentation de l'expression du gène Fshb dans l'hypophyse et une augmentation de la concentration sérique de FSH, 18 heures après l'injection et cela uniquement chez la femelle (Figure 2A et 2B). L'expression hypophysaire du gène Lhb et le taux sanguin de $L H$ restent inchangés chez la femelle comme chez le mâle (Figure 2A et 2B). L'administration d'AMH n'affecte pas les niveaux circulants d'oestradiol (ni de testostérone chez le mâle) suggérant que les modifications observées proviennent d'un effet direct sur l'hypophyse et ne sont pas la conséquence d'une modification de l'activité ovarienne.

Ces résultats établissent donc que l'AMH est capable d'agir in vivo sur l'activité gonadotrope hypophysaire en stimulant la synthèse de FSH chez la femelle sans affecter la LH. Cet effet pourrait être impliqué dans le dimorphisme sexuel de l'activité gonadotrope hypophysaire dans la période infantile qui se traduit par une plus forte sécrétion de FSH chez la femelle que chez le mâle [11]. Les mécanismes mis en jeu dans cette élévation de FSH, essentielle à la mise en 

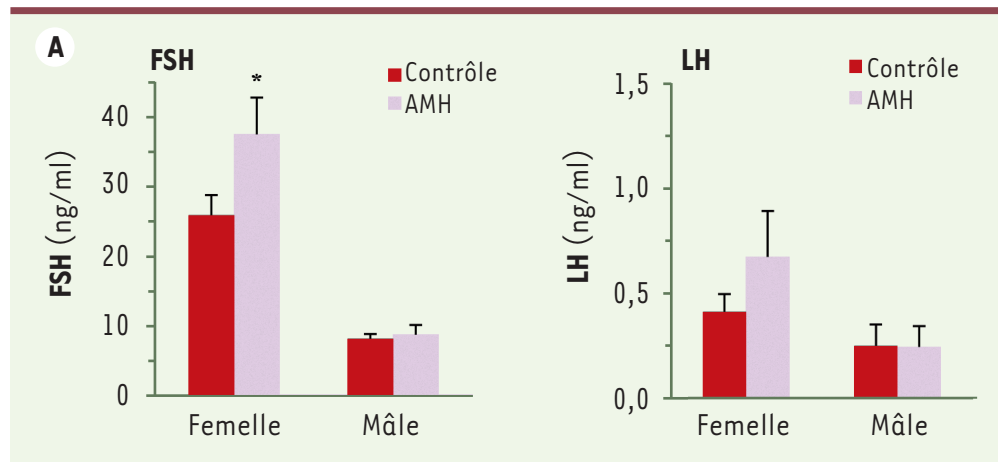

Figure 2. L'hormone anti-müllérienne (AMH) stimule, in vivo, la sécrétion de FSH et l'expression du gène Fshb chez le rat femelle immature mais pas chez le mâle. Une injection intrapéritonéale de $100 \mu \mathrm{l}$ d'une solution de sérum physiologique contenant ou non le précurseur de I'AMH $(10 \mu \mathrm{g})$ a été administrée à des rats mâles et femelles au $17^{\mathrm{e}}$ jour postnatal. Les concentrations sériques de FSH et LH (A) ainsi que les niveaux d'expression hypophysaire des gènes Fshb et Lhb (B) ont été déterminés 18 heures après l'injection.

\section{B}
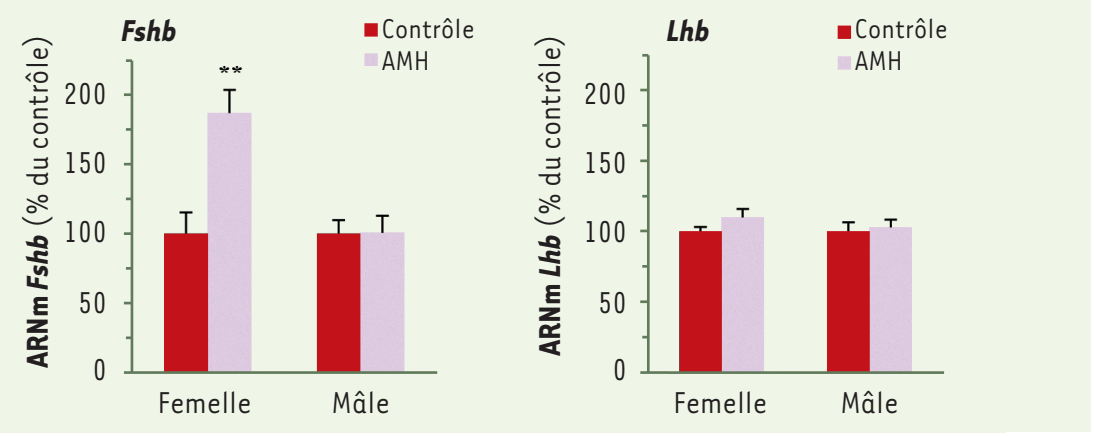

Chaque valeur représente la moyenne \pm SEM de 13 rats. $* P \leq 0,05 ; * \star P \leq 0,01$ par rapport aux rats contrôles. FSH : follicle-stimulating hormone ; LH : luteinizing hormone ; Fshb : follicle stimulating hormone beta subunit; Lhb : luteinizing hormone beta subunit; SEM : erreur standard à la moyenne.

place de la fonction de reproduction de la femelle, restent encore mal connus. En faveur d'un rôle important de l'AMH dans la fonction gonadotrope, nous avons également observé que la $\mathrm{GnRH}$, régulateur majeur des cellules gonadotropes, diminue les niveaux des transcrits Amhr2 et de la protéine AMHR2, in vivo chez le rat femelle de 18 jpn ainsi qu'in vitro sur les cellules L $\beta T 2$. Un réseau complexe d'interactions semble donc se mettre en place entre l'AMH, d'autres membres de la famille TGF $\beta$ et la GnRH pour réguler finement la synthèse de FSH.

Le défi sera maintenant de déterminer si des altérations du système AMH dans I'hypophyse peuvent être associées à des désordres de la puberté ${ }^{2}$. Anti-Müllerian hormone:

a new regulator of pituitary gonadotrope cells. Involvement in sexual dimorphism of gonadotrope activity before puberty

${ }^{2}$ Davantage de détails sont disponibles dans notre étude publiée dans Scientific Reports [12].

\section{LIENS D'INTÉRÊT}

Les auteurs déclarent n'avoir aucun lien d'intérêt concernant les données publiées dans cet article.

\section{RÉFÉRENCES}

1. Josso N, Picard JY, Rey R, di Clemente N. Testicular anti-Mullerian hormone: history, genetics, regulation and clinical applications. Pediatr Endocrinol Rev $2006 ; 3: 347-58$.

2. Lebeurrier N, Launay S, Macrez R, et al. AntiMullerian-hormone-dependent regulation of the brain serine-protease inhibitor neuroserpin. J Cell Sci 2008 ; $121: 3357-65$.

3. Wang Py, Koishi K, McGeachie AB, et al. Mullerian inhibiting substance acts as a motor neuron survival factor in vitro. Proc Natl Acad Sci USA $2005 ; 102$ : 16421-5.

4. Wittmann W, McLennan IS. Anti-Mullerian hormone may regulate the number of calbindin-positive neurons in the sexually dimorphic nucleus of the preoptic area of male mice. Biol Sex Differ $2013 ; 4$ : 18.

5. Bedecarrats GY, O’Neill FH, Norwitz ER, et al. Regulation of gonadotropin gene expression by Mullerian inhibiting substance. Proc Natl Acad Sci USA 2003 ; 100 : 9348-53.

6. Wang Py, Protheroe A, Clarkson AN, et al. Mullerian inhibiting substance contributes to sex-linked biases in the brain and behavior. Proc Natl Acad Sci USA 2009 ; 106 : 7203-8.

7. Cimino I, Casoni F, Liu X, et al. Novel role for antiMullerian hormone in the regulation of $\mathrm{GnRH}$ neuron excitability and hormone secretion. Nat Commun $2016 ; 7: 10055$.

8. Kim T, Do MH, Lawson MA. Translational control of gene expression in the gonadotrope. Mol Cell Endocrinol 2014 ; 385 : 78-87.
9. Counis R, Laverriere JN, Garrel G, et al. Gonadotropinreleasing hormone and the control of gonadotrope function. Reprod Nutr Dev 2005 ; 45 : 243-54.

10. Bernard DJ, Fortin J, Wang Y, Lamba P. Mechanisms of FSH synthesis: what we know, what we don't, and why you should care. Fertil Steril 2010 ; $93: 2465-85$.

11. Ojeda SR, Andrews WW, Advis JP, White SS. Recent advances in the endocrinology of puberty. Endocr Rev $1980 ; 1: 228-57$.

12. Garrel G, Racine C, L'Hote D, et al. Anti-Mullerian hormone: a new actor of sexual dimorphism in pituitary gonadotrope activity before puberty. Sci Rep $2016 ; 6: 23790$.

13. Catteau-Jonard S, Dewailly D, Prévot V, et al. L'hormone anti-müllerienne: une hormone ovarienne exerçant une rétroaction hypothalamique? Med Sci (Paris) 2016; $32: 441-4$.
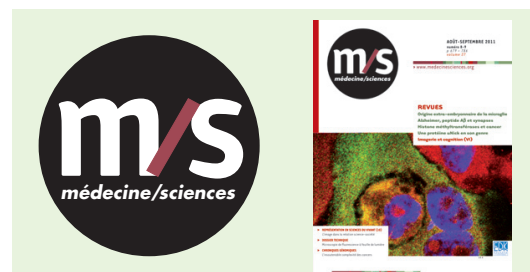

Abonnez-vous à médecine/sciences

Bulletin d'abonnement page 1148 dans ce numéro de $\mathrm{m} / \mathrm{s}$ 